\title{
FUGA DE VENEZOLANOS DURANTE LA REVOLUCIÓN BOLIVARIANA (1998-2007) ${ }^{1}$
}

\author{
Inés Guardia Rolando \\ Universidad Panamericana \\ Guadalajara (México)
}

\section{RESUMEN}

Tras la victoria electoral de Chávez, en 1999, una cantidad de venezolanos ha abandonado el país en busca de oportunidades. Los motivos abarcan desde temores por el destino político, hasta razones personales asociadas con su bienestar económico. El propósito de este ensayo es utilizar algunos postulados de los enfoques políticos y antropológicos, como construcción de identidades culturales y procesos de asimilación e integración, y aplicarlos a los emigrantes que residen en distintas ciudades de Norteamérica. Se maneja el siguiente supuesto, mientras los inmigrantes ejercen una actividad política que los mantiene pendientes de los acontecimientos políticos de su país de origen, más difícil resulta su proceso de integración al nuevo destino. El análisis se inscribe en la corriente denominada «historia de los hechos recientes» y combina la metodología histórica con categorías de las ciencias sociales, antropológicas y politológicas. El artículo ese organiza en dos apartados: el primero presenta un breve inventario de los grupos constituidos, se revisan sus fundamentos políticos y misión como actores políticos transnacionales. El segundo evalúa la posibilidad de que estos grupos se asimilen o si yace en ellos una resistencia a integrarse, es decir, si conservan su propia identidad sin articularse con los otros, comportándose como una minoría.

Palabras clave: Grupos de oposición, Inmigración, Asimilación.

\section{ABSTRACT}

After Chávez won the elections in 1999, huge amounts of Venezuelan have left the country looking for opportunities. The reasons are starting from fear to the political destiny, to personal reasons referring to economic issues. The main idea on this essay it's use

1 Este trabajo forma parte de una investigación en curso que centra su interés en distintos aspectos de la Revolución Bolivariana en Venezuela (1998-2007) conducida por la autora de este artículo. Una versión resumida de esta temática sin publicación fue presentada en el XXX Encuentro de RNIU celebrado en Metepec-México entre el 17 y 19 de octubre de 2007.

Fecha de recepción: 4 de febrero de 2008. Fecha de aceptación: 17 de junio de 2008 . 
some postulates focusing in politics and anthropologies, such as cultural background and process of assimilation and integrity and apply them to the emigrants that they are now residents in North America. What happens is that when the emigrants keep and active political relation with their original country while there are living some where else it results hard to them to get use to their new destiny. The essay fallows a current known as «History of recent acts» and combines a methodology with categories of social science, anthropologies and politics. The article its organize in 2 different sections: a) presents a short inventory of strength actual groups, we've check their political fundaments and their mission like active transnational politic actors. b) The second one analyze the possibility of this group to be successful while integrating, in other words, if it conserve his own sense with out depending of other, behaving like a minority.

Key words: Opposition group, Immigration, Assimilation.

\section{Introducción}

6 de cada 10 jóvenes no ven un futuro económico digno en la nación, $45 \%$ de los venezolanos quiere abandonar el país (Camel Anderson en: El Universal, martes 12 de agosto de 2003).

Fuera del país continúan también las manifestaciones por la situacion venezolana. El pasado sabado 18 de enero un grupo de aproximadamente 300 venezolanos realizó una marcha en contra el gobierno de Chavez en San Francisco, California. La marcha terminó en la plaza Bolívar de Fulton Street, cerca del Civic Center de San Francisco (www.eluniversal.com 20/01/2003).

Tras la victoria electoral de Hugo Chávez a la presidencia en 1999, una gran cantidad de grupos venezolanos pertenecientes a estratos económicos medios y profesionales se plantearon la necesidad de migrar hacia Estados Unidos de Norteamérica en virtud de que temían se tomarán medidas que limitaran el ejercicio de sus derechos ciudadanos y que afectaran sus intereses económicos².

Sus dudas en cuanto al destino político del país se basaban en la posibilidad de una eventual implantación de un régimen autoritario parecido al cubano, mientras que las razones personales asociadas al bienestar económico de sus familias se atribuían al alto índice de desempleo, elevada inflación, devaluación y controles de cambio impuestos por el gobierno ${ }^{3}$.

A partir del 2002 cuando los actores políticos domésticos vieron fortalecida su lucha en contra del gobierno, diversos grupos de emigrantes comenzaron a promover la participación política en contra del presidente Chávez desde sus nuevas trincheras, es decir, surgieron grupos de oposición al gobierno venezolano en algunas ciudades del mundo.

Cabe destacar que cuando las acciones del gobierno se tornaron más fuertes en contra de los opositores a partir de los acontecimientos políticos de abril de 2002, un gran número

2 Se utilizarán como sinónimos Estados Unidos, Norteamérica, el país del Norte y Estados Unidos de Norteamérica.

3 Según la encuesta de Consultores 21 de septiembre de 2003 el desempleo es del orden del 20.3\%, el empleo informal del $22.1 \%$, la inflación del $27.1 \%$ y la devaluación desde 1999 hasta la fecha es de $237 \%$. 
de activistas se vio en la necesidad de pedir asilo político ${ }^{4}$, muchos de los cuales promovieron este tipo de asociaciones. Lo anterior da la pauta para hablar en este momento del nacimiento de una emigración inusual para el caso de Venezuela.

El propósito fundamental de este ensayo es utilizar algunos postulados del enfoque político y antropológico como la construcción de identidades culturales y procesos de asimilación, integración y aculturación y aplicarlos a diferentes grupos de oposición venezolanos existentes en Estados Unidos de Norteamérica.

Se parte del siguiente supuesto de investigación mientras los inmigrantes ejercen una actividad política que los mantiene pendientes de los acontecimientos políticos de su país de origen, más difícil resulta su proceso de integración al nuevo destino.

Aunque se conocen las distintas clasificaciones de los inmigrantes —refugiados, asilados, inmigrantes, ilegales e indocumentados- y se reconoce su importancia. A lo fines de este ensayo se entiende la inmigración como el desplazamiento por diversos motivos de hombres y mujeres de un país a otro.

En tal sentido el ensayo puede entenderse como un intento por diferenciar las migraciones transitorias de las permanentes y las de motivación económica y de las políticas tal como lo recomendaba Léilo Marmora en su artículo «Desarrollo sostenido y políticas migratorias: su tratamiento en los espacios latinoamericanos de integración» (1994: 52).

Si bien es cierto que existen gran cantidad de grupos que apoyan al Presidente Chávez en el exterior como la Coalición de Chicago por Venezuela, el Círculo Bolivariano «Amada Libertad», Círculo Bolivariano de New York «Prof. Alberto Lovera», Círculo Bolivariano de Oregón, Comité de Solidaridad con Venezuela en Nueva York y el grupo Venezuela Solidarity por nombrar algunos de los más importantes, la prensa reporta que existen otros diecisiete círculos han sido creados desde Connecticut hasta Wyoming. En este ensayo se hará referencia únicamente a los que adversa la gestión del gobierno actual.

El análisis que se presenta se inscribe en la corriente historiográfica denominada «historia de los hechos recientes», la cual utiliza la categoría de actor político a fin de explicar el surgimiento y la acción de estos nuevos grupos, y combina la metodología propia de la disciplina histórica con categorías de las ciencias sociales, antropológicas y politológicas.

Con relación a la recolección de la información, se realizó una exhaustiva revisión del material hemerográfico correspondiente, así como un análisis de los documentos y páginas web de las asociaciones civiles o grupos de oposición residenciados en el exterior.

El artículo está organizado en dos apartados:

El primero presenta un breve inventario de los grupos constituidos; revisa sus fundamentos, su misión como actores políticos y hace énfasis en la teoría de los nuevos movimientos sociales, específicamente en lo que se refiere a la construcción de la identidad (Giddens, 1991; Touraine, 1992; Calhoun 1995; Castells, 1997).

El segundo apartado utiliza los conceptos de integración y asimilación según la connotación propuesta por Fernando Mires con respecto a entender que la integración es una necesidad, no obstante, que la asimilación es una opción.

Así pues, en este ensayo se asume que «integración implica conservar la propia identidad, pero en articulación con otras. La asimilación implica, en cambio, el abandono de la identidad propia en función de otra» (Mires, 2001: 114) y evalúa si es posible que estos grupos se asemejen a determinados modelos multiculturales prevalecientes en Estados Unidos, o si yace en ellos una resistencia a integrarse, es decir, a conservar su propia identidad articulándose con los otros; en tal sentido se estarían comportando como una minoría.

4 Para mayor información sobre las motivaciones $c f r$. Las estadísticas del Buró estadounidense de ciudadanía y Servicios de Inmigración (BCIS); Cuba encuentro.com, 15 de octubre 2003. 
Finalmente, se ofrecen las conclusiones y las fuentes utilizadas para la elaboración de este trabajo.

Así la primera tarea fue elaborar el inventario de los grupos de emigrantes venezolanos opuestos al gobierno que preside Hugo Chávez Frías (1999-2004) que se encontraban establecidos en diversas ciudades de los Estados Unidos de Norteamérica para explicar la importancia política de este actor en el contexto de la lucha por convocar al proceso electoral del referéndum revocatorio de 2004.

\section{Inventario de grupos de venezolanos en el exterior ${ }^{5}$}

La actual mecánica de la migración internacional —uno de los fenómenos globales más intensos y desafiantes de nuestra época-, ha mostrado signos de creciente complejidad. La migración no sólo es dinámica por el movimiento geográfico de individuos que supone, sino porque constantemente cambian las fuerzas sociales, económicas, culturales y políticas que impulsan a tomar la decisión de migrar (Santibáñez, 2004: 9).

Un estudio reveló que entre 1980 y 1990 los emigrantes mexicanos y centroamericanos radicados en Estados Unidos se duplicaron y alcanzaron una cifra cercana a los 8.400.000. Durante este período se desarrollaron grandes crisis económicas, políticas y sociales, a las que se pueden atribuir la evolución del fenómeno migratorio. Sin embargo, el fenómeno migratorio en el caso de Venezuela ha sido muy distinto dado las óptimas condiciones sociopolíticas que imperaron en aquel entonces en este país.

En este sentido, hasta hace unos diez años la emigración era un fenómeno que se presentaba de manera muy esporádica pues los venezolanos consideraban que su nivel de vida futuro no estaba en riesgo. Sin embargo, a partir del ascenso de Hugo Chávez a la presidencia cada vez es más frecuente y cotidiano encontrar testimonios como el que se presenta a continuación:

Tengo 25 años y soy venezolana de pura cepa. Con mucho dolor pienso abandonar mi país que amo profundamente, pero la situación política es cada vez peor. Leo sus experiencias y es bastante duro, pero para lograr un futuro lo más probable es que nos marchemos, dejando nuestra querida tierra. Trataremos de llenarnos de fortaleza para saber sobrellevar lo que nos espera y de paciencia para enfrentar las situaciones futuras (BBC Mundo, 5 de marzo de 2004).

La primera en volar a Estados Unidos fue la hija mayor, Mercedes Pérez: «Porque su esposo, Francisco Noguera, quien trabajaba en el IPSFA, fue amenazado y acosado por presentar oposición a la situación que se vivía en el país. Se vinieron en diciembre de 2002, justo en la época del paro» (El universal, 13 de noviembre de 2005).

En efecto, el censo estadounidense del año 2000 registró oficialmente a más de 91.000 venezolanos que viven a lo largo y ancho de ese país mientras que un reporte de inmigración para el año 2003 ubica que son unos 600.000 los que residían en los Estados Unidos; es decir, la comunidad de residentes se duplicó en escasos tres años. De estos, alrededor

5 Es conveniente señalar que dada la cantidad y variedad de grupos de oposición en las diferentes ciudades de los Estados Unidos, en el presente ensayo se refiere a una muestra representativa de ellos. Para mayor información consultar las páginas web de Resistencia Civil de venezolanos en el Exterior (Recibes) presidida por Miguel Hernández Andara, Proveo, SAVE Venezuela y Lucha Democrática. 
MaPa 1. Migración venezolana a las distintas ciudades de Estados Unidos

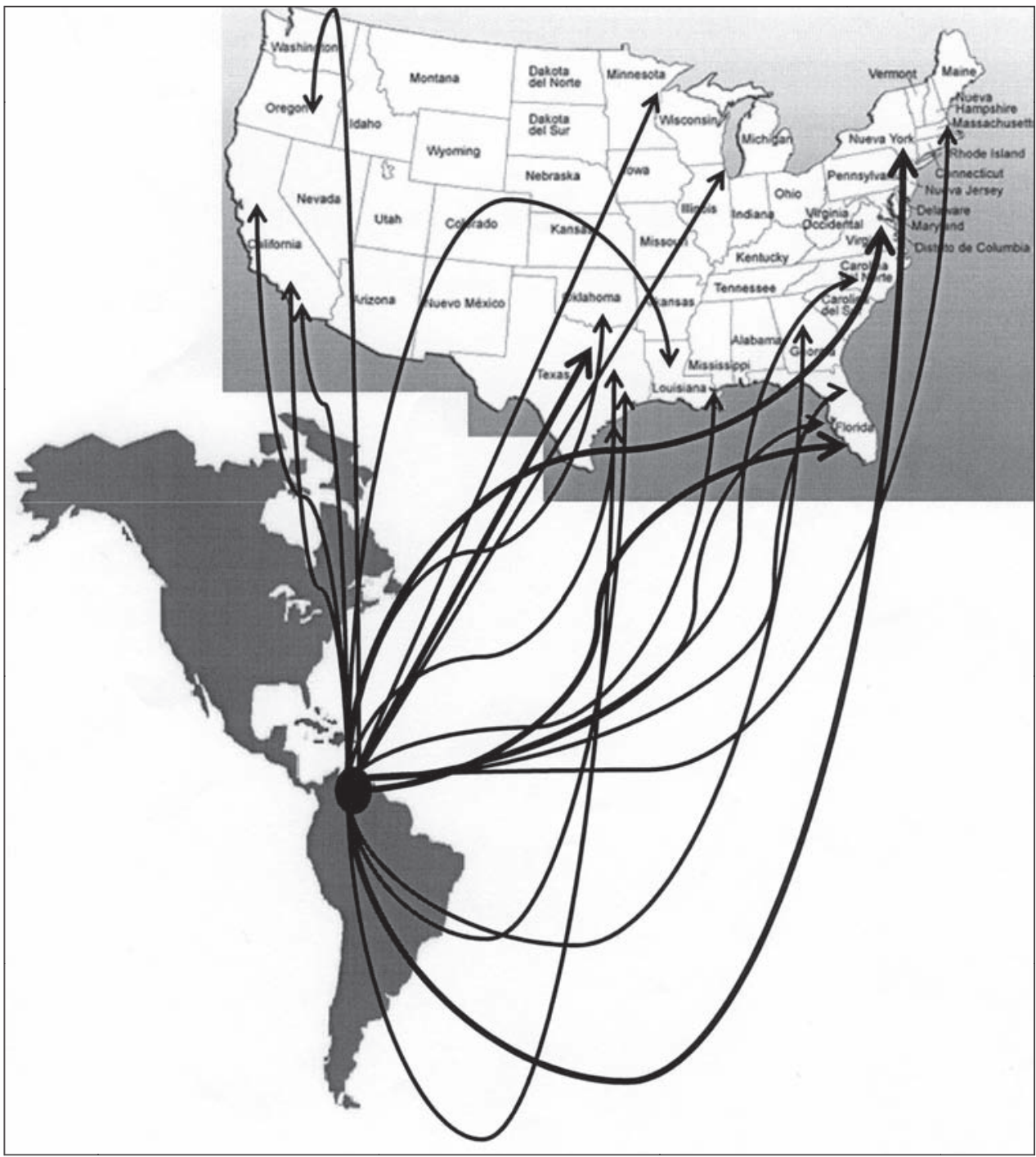

Elaboración propia.

de 400.000, están radicados en el sur del estado de Florida, y el resto concentrado en ciudades como Nueva York, Chicago, Los Ángeles y Atlanta (Red Venezolana, Sub comisión de relaciones de venezolanos en el exterior, Ocando, 24/09/2003).

El mapa $n^{\circ} 1$ muestra el flujo de migración de venezolanos hacia las distintas ciudades de Estados Unidos.

Las solicitudes de asilo de quienes se consideran perseguidos políticos hacia Estados Unidos, también ha aumentado en los últimos años, según cifras del Servicio de Inmigración 
y Ciudadanía (CIS). En el año fiscal 2002, los casos aprobados fueron de 155 y durante el 2004 subieron a 525, lo que representa, según el organismo, un incremento de más del $330 \%$ (El Universal, 22 de enero, 2004).

Estos nuevos residentes han invertido en bienes inmobiliarios, comerciales y residenciales; es común, sobre todo en Miami encontrar productos de la república bolivariana en diversos locales. Junto a ello se encuentran también distintos órganos divulgativos promovidos por actores domésticos y grupos de acción política de oposición al gobierno.

Algunos de los periódicos venezolanos que circulan en Estados Unidos son: Semanario Miami, el diario Venezuela al día, cuyo editor en Miami es Manuel Corao y La opinión, su editora política es Pilar Marrero.

Una de las Organizaciones civiles que en su momento congregó el mayor número de residentes en el exterior fue la Coordinadora Democrática, coalición de partidos de diferentes ideologías, asociaciones civiles y ONG, fundada en el año 2002 cuyo objetivo primordial fue dar a conocer al mundo las condiciones antidemocráticas que a su parecer imperaban en el país caribeño ${ }^{6}$. En ese mismo año, su dirigente en Miami Raúl Leoni explicó que diferentes grupos políticos había desplegado desde el 2000 un activismo para combatir el gobierno del presidente Chávez. (Cfr. Castro en: El Nuevo Herald, 22 de octubre de 2002).

Sin embargo, dicha oposición no sólo era señalada por los grupos y organizaciones civiles, sino que también diversos periodistas como Pilar Marrero, editora política de La Opinión sostenía que los venezolanos en Estados Unidos tendían a simpatizar con la oposición política que acusaba a Chávez de autoritario y de arruinar la economía nacional (New California Media, News Digest, 22 de diciembre de 2002).

Así, se puede considerar que, a pesar de las diferencias entre los objetivos de cada uno de los grupos, las agrupaciones analizadas en este ensayo coincidían en señalar su oposición al régimen presidido por Hugo Rafael Chávez Frías.

En este sentido, se puede afirmar que la llegada de Chávez al poder contribuyó al crecimiento del proceso inmigratorio de venezolanos. Es decir, su gestión gubernamental generó en el ciudadano de clase media y alta un sentimiento de amenaza en cuanto a la seguridad e integridad de sus intereses por lo cual optó por huir hacia otro país sin tomar en cuenta los obstáculos y dificultades que se presentan en todo proceso de integración en este tránsito.

Es conveniente señalar que dada la cantidad y variedad de grupos de oposición en las diferentes ciudades de los Estados Unidos, en el presente ensayo se refiere a una muestra representativa de ellos. Para mayor información consultar las páginas web de Resistencia Civil de venezolanos en el Exterior (Recibes) presidida por Miguel Hernández Andara, Proveo, SAVE Venezuela y Lucha Democrática.

Entre los grupos presentes en Estados Unidos se encuentran: la Coordinadora Internacional Venezolana con sede en Washington DC la cual se define como un grupo de ciudadanos residenciados en el exterior, organizados para defender el Estado de Derecho y el sistema democrático de Venezuela ${ }^{7}$; la Resistencia Civil de Venezolanos en el Exterior ${ }^{8}$, una aso-

6 Esta convergencia de partidos llegó a su fin en el 2004 al fracturarse internamente.

7 Su finalidad buscar «facilitar el intercambio de información entre la sociedad civil venezolana y el mundo internacional y promover y organizar medidas reactivas ante violaciones de los derechos humanos, la democracia y el Estado de Derecho en Venezuela». Pretenden «actuar como voceros de la sociedad civil en el extranjero; emitir opiniones profesionales ante los agentes extranjeros sobre la situación venezolana; crear una red de participación internacional de venezolanos y difundir en la prensa venezolana e internacional información relevante sobre la realidad de Venezuela» (http://www.civw.org).

8 La página web de está organización dice tener capítulos en las principales ciudades del mundo (www.11a. com/resistenciacivilexterior@yahoo.com). 
ciación civil fundada por el periodista zuliano Miguel Hernández Andara y Maria Teresa Van Der Ree para hacer sentir la voz de los venezolanos que viven fuera del país.

También se tiene la Federación de Asociaciones Venezolanas en Estados Unidos (FEDEAVEN), que agrupa diversas organizaciones comunitarias ${ }^{9}$. Este grupo espera ser la voz de los que llegan, y pretende convertirse en un grupo de cabildeo en Washington.

En relación a este grupo, sus organizadores consideran que FEDEAvEN constituye el primer esfuerzo en la historia migratoria venezolana a Estados Unidos por construir un ente central que coordine y abogue por las necesidades de sus nacionales desde la Florida, Nueva York y California, hasta Georgia, Texas y Luisiana.

«Es el primer indicador de que [los venezolanos] han venido para quedarse», afirmó el analista Sergio Bendixen, presidente de Bendixen \& Associates. «Cuando los éxodos llegan a este país no se organizan en los primeros años porque piensan regresar, pero cuando empeora la situación en sus países, comienzan a organizarse de esta forma» ${ }^{10}$.

No obstante, la creación de FEDEAVEN, cuyos miembros realizaron su primera convención en Miami durante el 2001, ha encontrado oposición por parte de la Hermandad Venezolano-Americana (Venamher), una de las asociaciones de conciudadanos más grandes de Estados Unidos con sede en Miami, la cual se ha negado a participar en el proyecto ( $E l$ Nuevo Herald, 2 de diciembre de 2001).

Llama la atención la noticia recogida por Marcelo Ballvé, quien asegura que «La brecha política que divide al presidente Hugo Chávez y la oposición venezolana también ha sembrado divisiones en las comunidades de inmigrantes venezolanos radicados en los Estados Unidos» (New California Media, News Digest, 22 de diciembre de 2002).

Adicionalmente, es posible identificar organizaciones que tienen relación con militares disidentes, tal es el caso de la Junta Patriótica Venezolana, bajo el liderazgo del capitán Luis García Morales ${ }^{11}$.

Existen otro tipo de agrupaciones asociados alrededor de temas como género tal como Mujeres por la Libertad, cuya vocera, Mariela Romero, desde Albany-Nueva York plantea que apoyan los esfuerzos reivindicativos de diferentes sectores de la sociedad venezolana.

Otra organización es la Fundación Venezolano Americana (FUNDAVEN) que se ocupa de tramitar ante la oficina de inmigración y otras instancias gubernamentales el estatus de protección temporal a algunos de los recién ingresados.

Ahora bien, entre las acciones que estos grupos han desarrollado, se encuentra la organización del proceso de recolección de firmas para el referéndum revocatorio, similar al que se realizó en Venezuela entre el 28 de noviembre y 1 de diciembre de 2003, con la finalidad de convocar el referéndum presidencial.

En Oklahoma, organizaciones como VENAO (Venezuelan American Association of Oklahoma) y RECIVEX (Resistencia Civil de venezolanos en el Exterior), junto a grupos de venezolanos residentes de este estado, organizaron dos eventos de recolección de firmas (Imagen Latinoamericana, Tulsa-Houston: semana del 3 al 9 de diciembre 2003).

9 Como la Casa Venezuela en Houston presidida por Manuel Barrios

10 Aspiran a buscar una solución al problema migratorio de decenas de miles de venezolanos que permanecen aquí ilegalmente. Además de velar por asuntos de inmigración, salud y empleo, se denomina como una entidad apolítica y sin fines de lucro. No obstante, la recién fundada federación en Miami, mantendrá un frente de alerta ante posibles atropellos contra las libertades individuales en Venezuela, según sus directivos (El Nuevo Herald, 2 de diciembre de 2001).

11 Según se lee en el artículo de prensa, «El objetivo: compartir información de contrainteligencia y jornadas de entrenamiento militar para estar preparados ante el peor de los escenarios, el de una posible guerra civil en Venezuela». Así pues, «Estamos dispuestos a prestar toda la ayuda que sea necesaria para enfrentar la tiranía de Chávez», dijo Rodolfo Frómeta, comandante del grupo radical (El Nuevo Herald, 22 de octubre de 2002). 
Asimismo en Nueva York y Florida, por mencionar sólo algunos lugares, se organizaron los representantes de «Venezolanos en el exterior» para recolectar las firmas, a pesar de que el Consejo Nacional Electoral decidiera no reconocer las que se recogieran en el exterior.

También se han producido manifestaciones de repudio contra la violencia y la represión chavista en diferentes ciudades de los Estados Unidos. Según se indica en El Universal se realizaron protestas en Miami, San Francisco, Nueva Orleans, Boston, Corpus Christi, Orlando, Austin, Atlanta, Charlotte, Dallas, Tulsa, Houston, San Diego, Tampa, Los Angeles y Minneápolis, así como en San Juan (Puerto Rico) tal como se describe en la siguiente cita textual:

En la zona noreste de Estados Unidos se eligió la ciudad de Washington, en el Distrito de Columbia, para realizar una demostración, justo frente a la Casa Blanca —en el 1600 de la avenida Pensilvania-, en repudio al gobierno de Chávez, a la que asistirán venezolanos residentes en los estados vecinos de la costa este de EEUU (El Universal, 9 de marzo, 2003).

En efecto, a finales de 2002, ante los excesos cometidos por el gobierno a través de los organismos de seguridad del Estado, a causa de las manifestaciones de ciudadanos por la negativa del Consejo Nacional Electoral (CNE) de reconocer las firmas del referéndum revocatorio presidencial, las organizaciones en el exterior, se movilizaron y protestaron ante las embajadas en contra de la violación flagrante de los derechos humanos.

Más recientemente, surgió la Organización de Venezolanos en el Exterior (ORVEX) compuesta por venezolanos residentes en Estados Unidos que se han sentido perseguidos al disentir del actual gobierno y por negarse a permitir que el ideal político de la Revolución Bolivariana — de corte Castro-Comunista, según sus organizadores- determine su futuro y el de sus hijos. Se identifican como una organización social y no política. Tiene tres objetivos fundamentales:

1. Asumir el desafió de abrir el camino para que cientos de miles de venezolanos que se encuentran en los EE.UU. como exiliados obtengan una protección temporal migratoria y una posterior residencia permanente por parte del Gobierno de los Estados Unidos.

2. Ofrecer un Centro de Apoyo Integral a los venezolanos, sobre todo a los recién llegados (cuyo numero aumenta día a día), para que se adapten al sistema americano.

3. Promover entre sus miembros los principios de solidaridad, servicio, convivencia mutua, y aquellos que nos permitan cristalizar la unidad de todos los Venezolanos en el Exilio con el propósito de lograr los objetivos propuestos (www.orvex.org).

Se plantean la unión de los venezolanos residentes en EEUU para lograr la protección temporal migratoria y una futura residencia permanente para aquellos que viven en el exilio, independientemente de su estatus legal.

Con relación a las agrupaciones anteriormente descritas, vale la siguiente reflexión: los fines de las organizaciones venezolanas y sus movilizaciones responden a motivaciones políticas y reaccionan ante acontecimientos que tienen su origen en el estado a diferencia por ejemplo de las asociaciones de inmigrantes mexicanos que han llegado a constituir un medio de reunión y unidad; incluso la clasificación de organizaciones que ofrece Palma Mora no contempla ninguna de carácter político. Según este autor 
las organizaciones de inmigrantes pueden clasificarse de la siguiente manera: asistencia social o beneficencia, educativas, religiosas, comerciales, socioculturales y recreativas (Palma, 2005).

A partir de los grupos estudiados, surgen varias advertencias analíticas que merece la pena resaltar: si bien las motivaciones iniciales para emigrar fueron diversas y distintas, una vez en el nuevo país cada uno de los inmigrantes se ha identificado con su compatriota, sin distingos de clases sociales.

Por otra parte, hay que señalar posibles errores en relación a las cifras de migrantes hacia los Estados Unidos pues no se conocen la forma cómo esta información fue recabada, en tal sentido debe tenerse precaución a la hora de elaborar generalizaciones.

Adicionalmente, la complejidad de la problemática analizada impone la necesidad de abordar el estudio de la migración y el surgimiento de grupos de oposición en el exterior desde la teoría de los nuevos movimientos sociales, en el sentido de que sus categorías hermenéuticas pueden ser utilizadas como concepto de partida para la realización de un estudio crítico que intente examinar las motivaciones de los actores.

Es decir, se busca entender estas nuevas formas de participación política desde la conformación y actividad que ellos ejercen. En tal sentido, nuestra atención se centra en los estudios que enfatizan en la conformación de los procesos de identidad ( $C f r$. Giddens, 1991; Touraine, 1992; Calhoun 1995; Castells, 1997), puesto que este abordaje teórico intenta comprender el porqué y el cómo del surgimiento de los nuevos actores sociales.

Finalmente, se debe considerar la posición sostenida por los multiculturalistas de origen marxista, para quienes el éxodo de venezolanos hacia los Estados Unidos no significaría problema alguno, pues de lo que se trataría es de ciudadanos sometidos al imperio de la ley participando en las conquistas de la civilización occidental, es decir: la democracia, el valor de la diversidad, el Estado de derecho, la libertad, la tolerancia y el pluralismo de los partidos. Según este enfoque no se persigue la integración puesto que se estaría contribuyendo a la multiculturalidad como proceso.

Tabla No. 1

SOLICITUDES DE ASILO POLÍTICO PRESENTADAS POR VENEZOLANOS EN EE.UU. (2000-2006)

\begin{tabular}{|c|c|c|}
\hline Año & Solicitudes & Aprobadas \\
\hline 2000 & 47 & 2 \\
\hline 2001 & 101 & 5 \\
\hline 2002 & 214 & 24 \\
\hline 2003 & 673 & 35 \\
\hline 2004 & 1623 & 59 \\
\hline 2005 & 1407 & 153 \\
\hline 2006 & 980 & 279 \\
\hline
\end{tabular}

Fuente: Aparisi (2007) EE.UU: «Asilo para venezolanos» en: BBCmundo, 27 de marzo. 


\section{Integración o asimilación de los grupos de venezolanos}

El incremento de la población hispana en Estados Unidos y su resistencia a una asimilación cultural plena, ha logrado el restablecimiento de la educación bilingüe, que fue duramente cuestionada por movimientos conservadores.

Según el Buró del Censo, la población hispana de Estados Unidos llegó a 35.300.000 personas en el año 2000, cifra que supera las 22.400.000 personas de 1990. De esta manera, los hispanos se ubicaron como la primera minoría del país, y su incremento del 58\%, superó el de otras minorías como los afroamericanos o los nativoamericanos (Últimas Noticias, domingo 18 de agosto de 2002).

En general, ha sido superada la etapa en la cual los gobiernos imponían la asimilación del extranjero ${ }^{12}$; hoy en día parecen imperar procesos de integración amparados y vigilados por el proyecto liberal del estado nación (Bauman, 2003: 111).

Es decir, los inmigrantes deben respetar la constitución y las leyes del país que los recibe y a cambio pueden conservar ciertos rasgos culturales y tradiciones asociados a su tierra natal en tanto no entren en conflicto con las normas y valores establecidos por la gran mayoría.

En el caso particular de los venezolanos emigrantes hacia los Estados Unidos, el análisis de la data muestra dos tendencias generales: los que van para quedarse y los que se fueron «mientras» mejoren las condiciones del país.

Entre los que toman residencia permanente en Estados Unidos figuran en los primeros lugares investigadores y académicos, que constituyen la llamada «fuga de talentos». La problemática de este sector se muestra en la siguiente cita textual,

El sociólogo Iván de la Vega, del departamento de Estudios de la Ciencia del Instituto Venezolano de Investigaciones Científicas, señala que aunque el Estado no ha recabado cifras, ha datos que pueden ilustrar la magnitud de la emigración en el área. Cita las estadísticas de la Nacional Science Foundation, de Estados Unidos, según las cuales 9000 venezolanos trabajan en esa nación en empleos vinculados con la actividad científica y tecnológica 'más de la mitad cuentan con la nacionalidad estadounidense y cerca de 3000 tienen visa permanente, lo que revela que casi en su totalidad se encuentran radicados en Estados Unidos'...(Núñez en: El Nacional, 23/11/2003:B-24).

Luego se tiene el grupo de los que recibieron asilo político. «Desde que llegó Chávez al poder, un total de 572 venezolanos han solicitado asilo por persecución política o por orientación sexual, de los cuales casi $20 \%$ ha obtenido respuesta afirmativa, mientras otro $40 \%$ está esperando por el Buró de Inmigración del Gobierno norteamericano» (Quinto Día, 15 al 22 de agosto de 2003).

Finalmente se encuentran los que se devuelven, entre los que figuran profesionales de las clases medias y altas, quienes una vez en los Estados Unidos, no han podido conseguir empleo en sus profesiones, y después de un lapso de espera considerable (entre un año y dos) y de ver mermados o acabados sus ahorros, regresan a su país en busca de mejores oportunidades.

12 A pesar de la tendencia a cercenar espacios multiculturales conquistados, como es el caso de la polémica ante la propuesta de prohibición del uso del velo y barba mantenida en Francia desde mediados de 2003 y que continúa en la actualidad. Recomendamos además revisar la tesis de Samuel Huntington, con relación a que los hispanos tienen una fuerte resistencia a la asimilación de la cultura estadounidense. 
Según un interesante estudio sobre la emigración venezolana realizado por la firma Datanálisis, se señala que

Las colonias de venezolanos en el exterior no sólo son numerosas, sino especialmente estructuradas. Localizaciones geográficas comunes como la de Weston, panaderías con cachitos, areperas con carne mechada, grupos cerrados de amigos venezolanos que se dan 'calor de familia', marchas y protestas en contra de Chávez frente al consulado, fiestas típicas con tequeños, cerveza Polar y hallacas navideñas envueltas en papel de aluminio, son sólo parte de la nueva realidad de ese grupo social que representan «los venezolanos en el mundo»... (León en: $E l$ Universal, 9/11/2003:1-17).

A pesar de que puede haber diferencias en la composición de los grupos de venezolanos en el exterior, éste no parece ser problema para las organizaciones de oposición, sino más bien el problema de legalidad/ilegalidad por problemas de visado.

Así mismo, a pesar de mantener un interés por los problemas domésticos, pareciera que uno de los objetivos para los cuales han servido estos nuevos actores sociales es para comportarse como un grupo con identidad colectiva, formando un nuevo tipo de «comunidad natural» (Bauman, 2003), lo que les permite mantener un tipo de prácticas, comunicación y discurso que les afianza los lazos identitarios como venezolanos, más allá de cuestionar la organización y estructura sociocultural de los norteamericanos.

En este sentido, y a pesar de que los venezolanos como grupo de potenciales inmigrantes hacia los Estados Unidos está en ascenso numérico, (ver mapa $\mathrm{n}^{\mathrm{o}} 1$ ) en ningún momento puede considerarse la posibilidad remota que implica un cuestionamiento al Melting Pot norteamericano, pues entre los objetivos de los grupos de oposición tomados como casos de estudio, no está planteado constituirse como un grupo de presión a favor de los derechos de los latinos; por lo menos, por ahora.

En otro orden de ideas, también es importante resaltar que tampoco es posible considerar que estos nuevos actores políticos se comporten como una minoría en el sentido propuesto por Paech (1998), puesto que ni siquiera poseen la ciudadanía de la mayoría y se mueven en la débil frontera de la legalidad/ilegalidad, anteriormente explicada.

\section{Consideraciones Finales}

El caso de la reciente inmigración venezolana se presenta como atípica, pues en nuestro caso, este fenómeno no ocurría desde los años 50 , cuando por razones de la dictadura militar presente en el país, un grupo minoritario de activistas políticos estaba en situación de asilo en distintos lugares.

Con respecto a la teoría de construcción de identidades, se aprecia que ella no se aplica en el caso estudiado, pues todavía no ha surgido una identidad nueva en quienes residen en los Estados Unidos. No obstante, se considera que el grupo de inmigrantes se ha integrado tal como lo plantea Mires, pues vive articulado a la otra cultura que lo rodea. Así pues, se puede afirmar que la asimilación por lo tanto no se ha producido.

La reflexión que se elabora, luego de observar la actitud de los venezolanos en el exterior, parte de la siguiente premisa: de variar las condiciones políticas y económicas del país, es probable que un alto porcentaje de aquellos que emigraron, regresen; pues estas causas se han presentado como el motivo de su migración.

Asimismo, es posible afirmar que la existencia de distintas organizaciones que desde los Estados Unidos están haciendo actividad política con repercusión en Venezuela, no repre- 
sentan un impacto en la cultura política norteamericana, pues su función ha sido más bien la de crear una identidad comunitaria entre los venezolanos de reciente inmigración, lo que ha permitido fomentar lazos de unión y solidaridad dentro de este nuevo grupo societal.

\section{Fuentes utilizadas}

BAUMAN, Zygmunt (2003). Comunidad. En busca de seguridad en un mundo hostil. Siglo Veintiuno Editores, España.

BAUMAN, Zygmunt (2001). La sociedad individualizada. Cátedra, Madrid.

BAUMAN, Zygmunt (2001). En busca de la política. Fondo de Cultura Económica, Buenos Aires.

MELUCCI, A. (1994): «¿Que hay de nuevo en los nuevos movimientos sociales?» en: Los Nuevos Movimientos Sociales: de la ideología a la identidad, E. Laraña y J. Gusfield (eds), Centro de Investigaciones Sociológicas, Madrid.

MIRES, Fernando (2001). Civilidad. Teoría política de la posmodernidad. Editorial Trotta, Madrid.

PIÑANGO, Ramón (2003). «Muerte de la armonía», En: Esta Venezuela, Ed. IESA, Caracas, pp. 17-28

SARTORI, Giovanni (2001). La sociedad multiétnica. Pluralismo, multiculturalismo y extranjeros. Taurus, Madrid.

SENTÉ, Richard (2001). «La calle y la oficina: dos fuentes de identidad» en: En el límite. La vida en el capitalismo global. Tusquets-Kriterios, Barcelona.

TILLY, Charles (1995). «Los movimientos sociales como agrupaciones históricamente específicas de actuaciones políticas», Sociológica, 28, 10, Mayo-Agosto, pp. 19-20.

www.eud.com

www.elnacional.com

www.globovision.com

www.miamiherald.com

www.civw.org (Coordinadora Internacional Venezolana)

www.venezuela-california.8k.com (Venezuela en el Norte de California) www.eluniversal. com/2003/01/20 